\title{
La información personal en la era digital
}

\author{
Javier TARANGO \\ tj.88888@hotmail.com \\ Universidad Autónoma de Chihuahua, México \\ www.javiertarango.com \\ Jesús LAU \\ jlau@uv.mx \\ Universidad Veracruzana, México \\ www.jesuslau.com
}

Recibido: 15/06/2013

Aceptado: 24/06/2013

\section{RESUMEN}

En el impulso de la sociedad del conocimiento la información personal es todavía un asunto pendiente, un campo inexplorado por la bibliotecología y las ciencias de la información. No existe, desde la perspectiva epistemológica, una teoría que explique sus principios o cualidades, una tipología que clasifique los documentos de carácter personal ni un método validado que nos permita comprender sus alcances en la era moderna de la información.

En este contexto, el artículo propone una serie de elementos metodológicos para la gestión de proyectos de información personal, recomienda aspectos prácticos para la organización documental a través de fundamentos bibliotecológicos tradicionales y diversas herramientas informáticas, además de teorizar sobre los retos que implica la aplicación de las propuestas de registro, almacenamiento, control y divulgación de la información personal en ámbitos profesionales, de investigación y académicos.

Palabras clave: Información personal; Administración de información personal; Gestión de documentos; Organización documental.

\section{Personal information in the digital age}

\begin{abstract}
Personal information has manifested itself as an unexplored field of library and information science, both from an epistemological and methodological perspective. Through this analytical-descriptive proposal, conceptual approaches are studied, showing a precise uncertainty about devoid of scientific terminology and accurate typology of personal documents.

Under such circumstances, the paper considers a number of methodological issues in the management of personal information projects, recommending practical aspects of documentary organization through traditional library science fundamentals and various computational tools, thus showing the need, benefits and challenges proposals involves applying for registration, storage, control and dissemination of personal information in professional, research and academic.

Keywords: Personal information; Personal information management; PIM; Document management; Documental organization.
\end{abstract}




\section{INTRODUCCIÓN}

La línea de análisis de este artículo se centra en la idea de que las personas que ejercen una profesión en ámbitos organizacionales, de investigación y académicos son generadores permanentes e ineludibles de documentos y deberán crear, mantener y evaluar diversos modelos para su almacenaje, localización y divulgación.

En los tiempos de la era digital, es necesario crear metodologías funcionales para el análisis de la información personal, que a su vez, permitan romper el paradigma del perfil profesional del científico de la información -más próximo a la gestión de procesos informativos y de innovación organizacional que al manejo de documentos personales- y proyectarlo hacia nuevas formas de ejecución y reconocimiento disciplinar.

Si bien es cierto que la sociedad del conocimiento demanda la formación de sujetos vinculados al acceso, uso y evaluación informativa (infocompetencias), pocas veces refiere la exigencia actual de que el sujeto genere dicha información y que trascienda el inexorable plano de consumo al utilizar elementos informativos que otros han creado.

Aunque la dinámica organizacional y los grandes escaparates multinacionales parecieran restarle importancia al proceso de información personal, es evidente el empuje y al mismo tiempo la incapacidad o la ausencia de sistemas para administrar sus recursos informativos ya que no saben priorizar aquellos documentos que contribuyen al desarrollo de sus actividades cotidianas.

La búsqueda de un sistema que permita administrar la información personal se ha dado especialmente entre las personas que generan y usan de forma regular recursos informacionales y no necesariamente bajo la iniciativa de bibliotecólogos y científicos de la información. Esto ha provocado que no exista un fundamento ni una metodología precisa para definir los procesos de administración de información personal.

Ante este escenario, los objetivos que se pretenden lograr son:

a) Identificar los principales conceptos sobre información personal desde diversas perspectivas (origen, uso y propósito).

b) Delimitar conceptualmente qué es la información personal.

c) Establecer lineamientos para definir las bases sobre proyectos de gestión informativa personal, tomando como referencia las necesidades particulares según los diversos ámbitos de acción profesional.

La metodología aplicada a esta investigación inició con la búsqueda de las referencias o literatura publicada sobre proyectos de información personal. Posteriormente se procedió a identificar aspectos metodológicos y propuestas de trabajo.

Las referencias que se encuentran en la red y otras fuentes bibliográficas sobre el tema son escasas, limitadas y en general muy vagas e imprecisas, solamente se hace alusión a una constante: la importancia de las tecnologías de la información y las 
comunicaciones (TIC) como herramientas fundamentales para el logro eficiente en el diseño y administración de proyectos de información personal.

Es importante considerar que las alternativas de administración de la información personal basadas en las TIC, se justifican en los ámbitos que se generan, ya que no existe otra propuesta proveniente de las ciencias de la información y documentación. En ningún caso se han considerado, por ejemplo, elementos básicos de la bibliotecología tradicional, cuya base permitiría la posterior mecanización de los procesos en dos sentidos: la generación de un sistema de información personal (repositorio) y la divulgación permanente de los contenidos personales a disposición del público en general.

Una preocupación latente luego de revisar la literatura y las referencias bibliográficas es la ausencia de una metodología específica para la administración de información personal. Las aproximaciones más directas versan en relación a los procesos de gestión, administración, diseño y actualización de los documentos personales, afrontando una problemática generalizada que conlleva a la imprecisión de elementos concretos tomando como referencia dos modalidades de trabajo:

a) La fragmentación, cuya propuesta establece que en la administración de información personal es necesario separar los documentos de acuerdo a tipos y propósitos, por tanto, se requiere trabajar con varios escenarios tecnológicos.

b) La no fragmentación, alternativa que pretende agrupar en una sola plataforma o escenario tecnológico o computacional los documentos personales, de tal forma que dentro de un mismo espacio se desarrollen clasificaciones específicas de los documentos, sin considerar como prioritario los propósitos por lo que fueron creados.

Es cierto que la información personal -ante las dudas que genera su imprecisa identificación y su falta de propuesta metodológica- debería considerar la adhesión a alguna disciplina científica en el ámbito de las ciencias de la información y documentación, antes de que se vea sólo como un tema relacionado con las TIC.

\section{CONCEPTUALIZACIÓN DE LA INFORMACIÓN PERSONAL: PANORAMA GENERAL}

Los limitados e imprecisos registros bibliográficos que existen sobre el tema de la información personal como proceso documental nos impiden compararlos sistemáticamente con los procesamientos de bibliotecas o centros de información general. Estos últimos funcionan a través de una serie de normas y reglamentos de valor global y parámetros operativos proyectados a un servicio público proveniente de infinidad de proveedores. 
En este sentido, se debe tomar en cuenta que la información personal nace como privada, por lo que se aproxima a la disciplina archivística, ya que está compuesta regularmente por documentos únicos (tipo manuscrito) y sus datos de identificación suelen ser mínimos.

Sin embargo, no son válidas todas las afirmaciones que relacionan estrictamente a la archivística como disciplina metodológica para el procesamiento de la información personal, ya que en principio no se ha tipificado lo que es un documento personal. La posibilidad de incluir documentos editados -aunque de forma estricta no entren en este rango- complementan los acervos de consulta individual.

Aunque la diferencia radica en la manera como la información se registra, divulga y es referenciada, todavía existe un vacío en la identificación tipológica de los documentos personales. Si estos son solo vistos como un producto documental entonces su clasificación dependería del formato en los que están creados (por ejemplo: documentos electrónicos y físicos) o por la intención de su contenido (artículos, cartas, tesis, cursos, etc.).

Finalmente si su taxonomía se basa en formas de divulgación se agruparían según el origen (propios no publicados y propios publicados, documentos de divulgación amplia y de divulgación restringida).

$\mathrm{Al}$ considerar que cada proyecto de gestión de documentos personales es diferente, es necesario distinguir expresiones como "información personal" y "datos personales". Los primeros son documentos que tratan cualquier temática y los segundos incluyen elementos aislados en el contenido que se consideran privados (incluso existen leyes de protección de los mismos).

Los datos que definen la información personal pueden clasificarse en esta lista: fecha de nacimiento, número de seguridad social, número de empleado en una organización, domicilio personal, etc. (Boardman, 2004). También comprende aquellos documentos o datos que se guardan en agendas personales como números de teléfonos, direcciones, número de cuentas bancarias -incluidos en algunos electrodomésticos que han conseguido actualmente automatizar este tipo de información documental (López Yepes, 2004).

Se entiende que los datos personales por su carácter confidencial no tienden a divulgarse y demandan un nivel de acceso restringido, sin embargo, desde que estos son depositados en una herramienta tecnológica se corre el riesgo de que pierdan privacidad y seguridad.

En Estados Unidos estos datos se conocen como "Personally Indetificable Information" e incluyen: número de seguro social, número de licencia de manejo y cuentas bancarias, sin embargo no considera: cuentas de correo electrónico o número telefónico (Narayanan y Shmatikov, 2010; McCallister, Graude y Scarfore, 2010). La divulgación de los datos personales tiende a restringirse, en cambio, la información personal tiende a consultarse y divulgarse (Boardman y Sasse, 2004; Boardman, 2004). 
Una de las primeras aproximaciones a la definición del concepto de información personal, vertidas por Barreau (1995), se refiere a la información almacenada dentro de un software de organización personal (desarrollada exprofeso o bien utilizando algún mecanismo comercial común a cualquiera que maneje una computadora). Estos documentos deberán estar concentrados dentro de los registros del mecanismo utilizado para almacenar y difundir la información personal de manera sistemática.

Podemos considerar que todo individuo posee información personal, pero mientras no esté organizada dentro de un sistema formal, no se puede hablar de un proceso de gestión. Los documentos considerados en el sistema deberán estar estructurados a través de categorizaciones que puedan facilitar la recuperación en cuanto sea necesario, tanto por el dueño como por aquellos sujetos interesados (Bellotti, et al, 2003).

Otras definiciones sobre información personal: aquellas que consideran incluir documentos identificados como de uso diario o cotidiano (Teevan, Jones; Bederson, 2006, Jones y Teevan, 2007), depositados de forma electrónica en una computadora (Katifori, et al, 2008), bajo diversas aplicaciones como procesadores de texto, administradores de direcciones y elementos de internet.

El sentido de la administración de la información personal a través de un sistema debería ser la divulgación -el individuo es consciente y desea extender su información a determinada área pública-. Esta gestión comprende toda información almacenada dentro de un sistema sin considerar aquella incluida en una herramienta específica.

El personal académico y de investigación, así como estudiantes o profesionales de la información, pretenden crear un espacio que administre sus documentos, especialmente cuando la información está relacionada con los contextos laborales en los que se desempeñan (Barreau y Nardi, 1995).

La administración de la información personal es un término que comprende la recolección, almacenaje, organización y recuperación de documentos, principalmente en formato digital (tales como: correos electrónicos, archivos, citas, recordatorios, contactos, libreta de direcciones o bookmarks, etc.) (Landsdale, 1988). Esta clase de elementos pueden considerarse como datos personales y de carácter privado, así como de uso propio, apoyo al trabajo y sustento a las necesidades del usuario en su tiempo libre (Bergman et al, 2004).

Otro aspecto importante en los procesos de gestión informativa personal es el lugar en donde se deposita. Autores como Collins y Kay (2008) consideran a la computadora personal como el mecanismo típico para su almacenaje y divulgación, por tanto, los documentos -tanto en formato electrónico o físico- que son considerados personales, pero no de la propiedad del sujeto, requieren referenciarse en el sistema indicando únicamente el vínculo para su localización en el lugar donde el editor los resguardó.

Una vez que exista el medio para depositar los documentos personales, el límite de acceso dependerá del enfoque sobre sus formas de interacción, mismas que pueden suceder: dueño-documentos o bien, documento-público en general (Katifori, et al, 
2008). El dueño de los documentos personales determina el alcance que se tendrá en la divulgación de los mismos.

La terminología revisada tipifica este concepto como "información personal", la única definición encontrada en español se refiere a "documentación personal", citada por López Yepes (2004) en el Diccionario Enciclopédico de las Ciencias de la Documentación, incluye todos los tipos de documentos que se emplean de manera particular en el trabajo, las relaciones sociales o en casa y propone un ordenamiento sencillo y que represente un valor para quienes los emplean (sin determinar si es sólo el dueño o diversas personas quienes disponen de la información).

De acuerdo a las distintas vertientes encontradas en el proceso de revisión documental para definir la información personal y tener un concepto preciso que nos permita acercarnos analíticamente a sus elementos y funcionamiento, creemos que lo esencial es integrar una serie de propuestas que clasifiquen los documentos personales, definir los límites de acceso y divulgación, determinar origen, uso y propiedad de los documentos y los mecanismos para identificarlos, ordenarlos y almacenarlos.

\section{LÍMITES EN LA INFORMACIÓN PERSONAL: DEL HABEAS DATA (DATOS PERSONALES) A LOS ASPECTOS PÚBLICOS}

El tema de la información personal muestra diversas aristas, enfoques de análisis que oscilan entre su origen y los mecanismos de control que imponen los dueños de los documentos de acuerdo a sus necesidades o niveles de restricción.

De acuerdo con Jones (2008), no existe un límite en la cantidad de información personal, pero definitivamente los proyectos de gestión de esta naturaleza suceden en individuos que han generado la suficiente para poder administrarla bajo una metodología o sistema.

Si bien en la cantidad de documentos no existe límite, en lo que se refiere a los niveles de divulgación sí lo hay ya que el dueño establece un parámetro de dominio sobre ellos. Algunas veces el control suele ser:

a) Voluntario. La información es exclusivamente de uso personal o permite a otros -de forma limitada- el acceso a ciertos documentos de carácter general.

b) Obligatorio. Se le conoce como habeas data (información muy personal). Lo establece la legislación como una modalidad de amparo para que todo individuo tenga el derecho de acceder a la información personal que le concierna, ya sea en archivos o ficheros de datos públicos o privados, con el fin de revisar y obtener informes sobre ella y, si es el caso, poder solicitar la suspensión, rectificación, confidencialidad o actualización (Navarro Jiménez, 2012).

Alguna información personal -relacionada con documentos que contengan datos personales- puede observar una condición dual: estar bajo pleno control de su dueño 
y al mismo tiempo, a disposición de otras personas u organismos. Esto significa, que aunque la información este a disposición de dos o más instancias, no necesariamente es divulgada por ninguna de las partes.

Otro aspecto de los límites que establece la información personal se observa en la movilidad natural o flujo informativo y se puede clasificar en dos tipos:

a) Información dirigida al dueño (se conserva y algunas veces ya no agrega valor) e

b) Información enviada por el dueño (ocasionalmente está provista de límites en los niveles de divulgación).

Anteriormente se mencionó que existe una inclinación al uso de la computadora para la gestión informativa (lo que significa invariablemente una limitación de espacio), sin embargo, otros autores como Berman et al. (2004), indican que habrá que superar esa barrera y no olvidar los elementos existentes en formato físico. Recomienda además dos aspectos: la posibilidad de convertirlos a formato digital o bien, generar mecanismos de referencia para su localización en otras fuentes.

Las limitaciones en la divulgación de la información personal -excepto aquella que contiene datos personales- no deberían pasar desapercibida en los tiempos actuales que demandan dinamismo al momento de divulgar y acceder a la información. Komninos, Baillie y Barrie (2008) consideran que la divulgación digital que no se circunscribe a un mero ordenador fijo y debería extenderse a los dominios o áreas de proyección tanto del profesional de la información como del usuario común.

Boardman (2004) considera que la información personal no tiene límites, por lo que es necesario integrarla en repositorios viables, permanecer en ordenadores móviles o en otras plataformas incluidas en la red de forma remota que contienen documentos relacionados con el medio social, personal y profesional del dueño.

Crear repositorios para almacenar la información provoca que sean considerados medios dinámicos de ingreso y descarte (Katifori, et al., 2008), otros los nombran silos de información (Collins y Kay, 2008) colecciones de documentos de mayor permanencia y en constante crecimiento. Se pueden incluir: información en la que trabaja el sujeto, datos científicos, correos electrónicos y colecciones de documentos en medios electrónicos, fotografías digitales, videos, música o documentos impresos digitalizados (Teevan, Jones, Bederson, 2006; Jones y Teevan, 2007).

De acuerdo a los diversos entornos de aplicación (social, personal y profesional) el sentido de la información personal - más que la simple acumulación- es el uso de los recursos y el control que el dueño de los documentos establece (niveles de acceso) (Bergman et al, 2004; Teevan, Jones, Bederson, 2006; Jones y Teevan, 2007). 


\section{INTEGRACIÓN DE PROYECTOS DE ADMINISTRACIÓN DE INFORMACIÓN PERSONAL}

Los proyectos de administración de información personal suelen tener ciertas complejidades ya que demandan la integración diagnóstica de necesidades particulares, por lo que dependen de diversas condiciones, entre las que se destacan las áreas de actividad laboral, los tipos de documentos y los formatos de presentación.

Ante esta condición -y de acuerdo a la revisión documental y al criterio empírico impuesto en este artículo- se debe considerar que la integración de un modelo de información personal debe respetar el principio de ciencia archivística conocido como "principio de procedencia" o "principio de respecto a la procedencia de los fondos", en donde se considera lo siguiente (Jones, et al., 2008):

a) Debe ser producto natural de la actividad propia del sujeto dueño de los documentos, por lo tanto, identifica al autor, su naturaleza, estructura y áreas de actividad.

b) Poseer su propia estructura interna, ejecutada por el dueño de los documentos o de quien los ha creado o acumulado.

c) Conocer los procedimientos administrativos del dueño de los documentos, así como su evolución histórica.

El principio de procedencia ofrece múltiples ventajas, entre ellas, considerarse como el marco conceptual de una tesis, lo cual implica cierta complejidad y se compone de cuatro actividades principales que deben dar como resultado una especie de prototipo de la clasificación de los documentos personales (Barreau, 1995):

a) Procedencia o mecanismo de adquisición de los documentos que forman la colección.

b) Organización documental de los mismos a partir de un criterio personal de agrupación de acuerdo a ciertas categorías.

c) Definición de las formas de mantenimiento del almacenaje de los documentos, identificando aquellos que deberán observar a largo o corto plazo de permanencia según sus características.

d) Formas de recuperación de los documentos para su uso posterior.

La definición del modelo deberá ser una representación formal de las ideas y necesidades conceptuales de la persona que lo crea y en las cuales se integren las clases y subclases de forma lógica. Autores como Sauermann, Elst y Dangel (2004), consideran que la estructura de los elementos del modelo puede proyectarse en tres dimensiones: 
a) Básica. Integra únicamente clases generales o superclases de otras clases.

b) Media. Incluye sub-clases de clases mayores por ejemplo: personas, proyectos, organizaciones, etc.

c) Alta. Define clases generales, sub-clases de cosas y puede llegar a aspectos específicos, por ejemplo, de personas-concepto, organizaciones-concepto, documentos-concepto, etc.

Sin importar la dimensión del proyecto -la cual propicia la complejidad del modelo- es ineludible aceptar el reto de crear un sistema taxonómico documental que defina clases y subclases de forma ordenada: mensajes (enviados, recibidos, etc.), artículos, personas, asociaciones, etc.

Aquí es cuando surge el problema de la eliminación de duplicaciones y la estandarización de las jerarquías que expresan las nomenclaturas y los niveles de subordinación (Dong, 2005).

Las taxonomías pueden presentar combinaciones cronológicas, de forma, tipo de documento, intención, etc., por ejemplo, en ámbitos académicos o de investigación, los aspectos cronológicos (períodos en que se produjeron los documentos) son fundamentales antes de la tipología de los documentos (artículos, libros, capítulos de libro, ponencias, etc.).

Para el diseño del mapa conceptual que describa los elementos del sistema de administración de información personal, se recomienda el uso de ontologías, las cuales especifican conceptualmente los principales componentes: clase, relaciones, funciones, axiomas e instancias (Lozano Tello, 2011).

La presencia de las ontologías tiene aplicaciones en múltiples campos del quehacer humano, en donde categorizan los elementos a ordenar, ejemplo de ello son: la Web semántica, procesos de gestión de conocimiento, e-learning y los elementos de la arquitectura de la información.

La elaboración de ontologías permitirá hacer mapas conceptuales de la estructura que observará la información personal. Este procedimiento permite integrar de forma abstracta, expresiva y flexible los contenidos del proyecto, de tal manera que se planeen repositorios de información en forma semántica, facilitando posteriormente la recuperación de información tomando como referencia los conceptos de orden del propio dueño del sistema.

Puede decirse que la ontología es un índice de palabras clave ordenadas de forma jerárquica. La adaptación semántica cobra especial importancia en la gestión de información personal, ya que el profesional involucrado deberá entender las condiciones precisas del usuario en cuanto a su conocimiento y organización, entendiendo, manejando y usando el cúmulo de información que recibe y almacena (Kratkiewicz y Mitchell, 2004).

Respecto al esquema estructural de los documentos - según Khoo, Luyt, Ee, Osman, Lim y Yong (2007)- deben considerarse los siguientes aspectos: 
a) Arquitectura de los archivos (forma de organización e identificación de los archivos por carpetas, etiquetas o categorías, nombre y tipos de archivos, estructuras jerárquicas, temporalidad de las carpetas, etc.)

b) Comportamiento del usuario (formas de crear, mantener y gestionar los archivos, así como la estrategia para localizar y recuperar información y documentos).

Un aspecto importante en la integración del sistema de información personal especialmente cuando se aplica el principio de No Fragmentación- es la definición del medio electrónico en donde se depositarán los documentos personales. Estos pueden mostrar un acceso directo o bien, representar ligas de localización que no están necesariamente en el sistema.

Por ejemplo, pueden citarse sistemas integrales que permiten la administración de información personal, tales como: SEMEX (SEmantic Explorer). Es un sistema de gestión informativa personal que ofrece a los usuarios una plataforma flexible que organiza los documentos y asociaciones entre las clases (Dong, Halevy, Nemes, Sigurdssin, Domingos, 2004). Este software permite diferenciar entre datos personales y datos públicos. Otra recomendación es QUILL, un sistema con interfaces que puede ser tomado en cuenta como referencia para desarrollar adaptaciones (Goncalvez y Jorge, 2008).

Después de precisar las categorías y subcategorías taxonómico-documentales, se procede al registro de los documentos específicos incluidos en cada una de ellas. En este sentido, se recomienda el uso de elementos de generación propia que faciliten la ordenación y la posterior localización de los documentos. Algunas recomendaciones prácticas son las siguientes:

a) Nombre asignado al documento. Se refiere al título abreviado o completo del nombre del documento, aunque suele ser extenso se recomienda el uso de numeraciones o bien prefijos, sustantivos, sufijos, fecha (año, mes, día), ejemplo: "PonBraIDICT 131119" (Ponencia Brasil IBICT 19 de noviembre de 2013), equivalente a (tipo de documento o prefijo: ponencia; lugar o sustantivo: Brasil: institución o sufijo: Brasil y fecha).

b) Elementos esenciales de la descripción-acceso al documento. Autor, título, tema.

c) Elementos esenciales de referencia. Autor, título, lugar, editorial, fecha.

d) Registro de metadatos (utilizar los elementos propuestos por el Dublin Core).

e) Orden para los libros impresos referenciados. Utilizando sistemas universales de clasificación (Decimal Dewey, Clasificación de la Biblioteca del Congreso, etc.).

f) Artículos de revistas y documentos. Utilizar cajas temáticas de agrupación cronológica. 
Una forma idónea de sustentar un modelo de información personal -recurriendo a los procesos de No Fragmentación- es la creación de un sitio web personal. Estratégicamente facilita las acciones de agrupación de documentos tipo repositorio, con poca o nula restricción de acceso. Además, permite la clasificación de documentos por categorías generales cronológicas (año de creación) y por subcategorías según el formato de los documentos: artículos, capítulos de libro, libros, materiales académicos, tesis, tesinas, trabajos de graduación, ponencias (presentaciones y en extenso), etc.

Infinidad de académicos utilizan este medio que les permite a estudiantes, miembros de su comunidad académica y al público en general poder accesar a determinada información y documentos.

Las plataformas recomendadas para administrar sistemas de información personal No Fragmentados son:

1) Joomla (http://www.joomla.org/) es un sistema de gestión de contenido (en inglés Content Management System o CMS) que permite desarrollar sitios web dinámicos e interactivos. Permite crear, modificar o eliminar contenidos de un sitio web de manera sencilla a través de un panel de administración. Es un software de código abierto. Puede utilizarse en una computadora local, en intranet e internet.

2) Drupal (http://drupal.org/) es un sistema de gestión de contenidos, modular y multipropósito, cuya confiabilidad permite publicar artículos, imágenes u otros archivos y servicios añadidos como foros, encuestas, votaciones, blogs, administración de usuarios y permisos. En lugar de almacenar sus contenidos en archivos estáticos -en el sistema de ficheros del servidor- fija el contenido textual de las páginas y otras configuraciones almacenadas en una base de datos y se editan utilizando un entorno web. Es un programa libre ideal para gestionar y construir comunidades en Internet.

La dinámica de cada modelo de administración de información personal puede variar de acuerdo al número de documentos que lo compongan, la frecuencia con la que se incorporan nuevos datos y el nivel de permanencia o ciclos de vida de los elementos que integran la colección (Boardman y Sasse, 2004).

Por esta razón es imprescindible definir inicialmente el esquema de agrupación documental y la asignación de nomenclaturas que permitan ordenar los datos de forma correcta. De esta forma no es necesario agregar nuevas categorías que hagan más complejo el sistema, sino simples subcategorías que mantengan la uniformidad en el crecimiento.

Cuando un sistema de gestión de información personal no puede mantener el principio de No Fragmentación ni agrupar todos los elementos en un solo sistema clasificatorio puede recurrir a la fragmentación (llevar colecciones diversas usando otros 
medios de administración documental), aunque más compleja, permite el uso de otros medios electrónicos para el registro de la información.

La aplicación del principio de Fragmentación considera las siguientes propuestas electrónicas de trabajo, en donde se recomiendan herramientas específicas y se describen sus características:

a) Seguimiento a sitios web. La herramienta recomendada es Delicius (http://www.delicious.com/), el cual es un sitio que combina los servicios de un bookmarking común en el que se tiene la oportunidad de albergar y dar seguimiento a las páginas web de interés. Funciona como una sencilla red social en la que se pueden compartir con otros usuarios que tengan los mismos gustos o intereses, tener acceso a los marcadores de otras personas e incluso ver los marcadores en la web dependiendo de las áreas de interés.

b) Administración de referencias. Se proponen dos herramientas: 1) Mendeley (http://www.mendeley.com/) una aplicación web y de escritorio gratuita que permite gestionar y compartir documentos de investigación, encontrar nuevos datos y colaboración en línea. Ofrece una aplicación de gestión de PDF y gestión de referencias y una red social online para investigadores; y 2) Tweetdeck (http://www.tweetdeck.com/) como aplicación de escritorio en Adobe AIR para Twitter, Facebook, LinkedIn, Google Buzz, Foursquare y MySpace. Como otras aplicaciones para Twitter, interactúa con la API de Twitter para permitir a los usuarios ver y enviar tweets y ver sus perfiles.

c) Servicio de mensajería electrónica. Twitter es el mecanismo más conocido (https://twitter.com/). Es un servicio de microbloging que permite enviar mensajes de texto plano de corta longitud de máximo 140 caracteres llamados tweets, mismos que se muestran en la página principal del usuario.

d) Fotografías. Se recomiendan dos herramientas de administración de documentos: 1). Flickr (http://www.flickr.com/) que es un sitio web que permite almacenar, ordenar, buscar, vender y compartir fotografías y video en línea. Cuenta con una oportunidad de usuarios que comparten las fotografías y videos creados por ellos mismos; y 2) Picasa (Picasa.google.com/intl/es/) como un organizador y visor de imágenes, además de permitir la edición de fotografías digitales. Adicionalmente posee un sitio web integrado para compartir fotos.

e) Colecciones de documentos (artículos, libros, etc.). Dspace (http://www.dspace.org/) es un software de código abierto que provee herramientas para la administración de colecciones digitales y comúnmente es usada como solución tipo repositorio institucional. Soporta una gran variedad de datos, incluyendo libros, tesis, fotografías, películas y videos, datos de investigación y otras formas de contenido. Los datos son organizados como ítems que pertenecen a una colección, cada colección pertenece a una comunidad. 
f) Música. Se recomienda iTunes (https://itunes.apple.com/). Es un reproductor de medios y tienda de contenidos multimedia desarrollado con el fin de reproducir, organizar y sincronizar música.

g) Videos. La herramienta más popular es Youtube (www.youtube.com) es un sitio web en el cual el usuario puede subir y compartir videos. Es muy popular gracias a la posibilidad de alojar videos personales de manera sencilla. Aloja una variedad de opciones: películas, programas de televisión y videos musicales. A pesar de sus reglas en contra de subir videos con derechos de autor, este material existe en abundancia, así como contenidos amateur como videoblogs.

h) Elucubraciones personales. Para administrar este tipo de información personal se recomienda usar Blog (es.wordpress.com/), el cual es un sistema de gestión de contenidos enfocado a la creación de blogs (sitios web periódicamente actualizados). Las causas de su enorme crecimiento son entre otras: su licencia, su facilidad de uso y sus características como gestor de contenido.

i) Mensajes de correo electrónico. Dos formas de administrar los correos electrónicos son Outlook y Thunderbird (https://www.mozilla.org/esES/thunderbird/). Es un proyecto de software libre y código abierto cuyo software ha sido usado para crear algunas de las mejores aplicaciones de Internet.

j) Contactos personales. Linkeln (mx.linkedin.com/) es un sitio web orientado a los negocios cuyo propósito es que los usuarios registrados puedan mantener una lista con información de contactos de las personas con quienes tienen algún nivel de relación llamado conexión. Puede usarse para lo siguiente: establecer redes de contactos favoreciendo la interactividad, los usuarios pueden subir su propio curriculum vitae o diseñar su propio perfil, funciona como directorio de recursos humanos, pueden subirse fotos, etc.

k) Administración de documentos. Una alternativa con múltiples aplicaciones, especialmente en ámbitos universitarios (i.e. revisiones de tesis, trabajos académicos, desarrollo de manuscritos para publicaciones, etc.), en donde se involucran una o más personas de forma interactiva en una misma actividad, ofrece múltiples alternativas interesantes entre las que se destacan:

1) Dropbox (https://www.dropbox.com) es un servicio gratuito que permite disponer de fotos, documentos y videos en cualquier parte. Esto significa que cualquier archivo que se guarde puede ser consultado en todos los equipos disponibles, tales como: computadoras, teléfonos y en el sitio web de esta plataforma. Este sistema permite compartir ítems con los demás, sin riesgo de perder documentos. 
2) Google drive (https://drive.google.com/start), es un servicio de almacenamiento de archivos en línea de reciente creación (2012) pasando de Google Docs a Google Drive. Cada usuario cuenta con cinco Gigabytes de espacio gratuitos para almacenar sus archivos, ampliables mediante pago.

3) Skydrive (https://skydrive.live.com/), plataforma que permite a los usuarios subir archivos de una computadora y almacenarlos en línea (nube), siendo posible acceder a ellos desde un navegador web o un dispositivo local. El servicio permite controlar el acceso a los archivos del usuario en donde los documentos se comparten públicamente. Cuenta con siete GB de almacenamiento gratuito y pueden subirse cinco archivos a la vez.

4) Slideshare (www.slideshare.net) es un sitio web que ofrece a los usuarios la posibilidad de subir y compartir a un público general o particular presentaciones de diapositivas en PowerPoint, documentos en Word, Open Office, PSD, Portafolios, etc. Proporciona la capacidad de evaluar, comentar y compartir el contenido subido. El servicio de alojamiento es gratuito y los usuarios pueden acceder registros.

\section{PRINCIPALES RETOS DE LA INFORMACIÓN PERSONAL}

La evolución temática de la información personal exige identificar los obstáculos que se derivan de la propia naturaleza de los documentos personales, lo que provoca la necesidad de impulsar o evitar su fragmentación (Jones, 2007).

Las necesidades de fragmentación informativa surgen principalmente al administrar colecciones de documentos personales ya que es necesario separarlos en distintos formatos (electrónicos o físicos) y tipos (textos, fotografías, música y videos) y muchas veces no se cuenta con estrategias o herramientas que ofrezcan soluciones integrales para la gestión de documentos.

Para integrar los documentos personales en un solo sistema de administración no Fragmentado, es necesaria la suma de todos los ítems previstos dentro de una colección general, por tanto, es imprescindible definir herramientas de alto alcance para la solución del problema.

Estas alternativas de solución pueden variar, sin embargo, deberán observar dos condiciones: la trasversalidad (cross-tools), de tal forma que separen los documentos por carpetas (Boardman, Sasse y Spence, 2002) y la acción longitudinal, en donde se recolectan las subcarpetas y con el paso del tiempo se van actualizando, en la medida que se incorporen nuevos documentos a la colección (Boardman y Sasse, 2004). Al cumplir ambas condiciones sucede: 
a) Una búsqueda eficiente en diversas bases de datos que componen el sistema de administración de la información personal.

b) La identificación de carpetas iguales en distintas colecciones, evitándose la duplicidad.

c) La creación y el agregado de nuevas carpetas sin afectar a las ya existentes.

Ante el limitado desarrollo de modelos no fragmentados de administración de información personal urge diseñar prototipos de sistemas cuyo fin será integrar documentos personales, según Boardman y Sasse (2004), en dos perspectivas clave:

a) Integración apoyada en el manejo de múltiples tipos de información dentro de las herramientas que comprende el prototipo.

b) Unificación e integración de múltiples tipos de información en una interface única, consolidando toda la tipología de documentos en un sistema integral.

Otro reto que enfrentan los procesos relacionados con la información personal es que una vez integrado el sistema debe mantenerse actualizada la colección, ya que ante el dinamismo en la generación de nuevos documentos, estos deberán incorporarse al acervo, así como descartar aquellos que no ofrezcan utilidad (Boardman y Sasse, 2004).

Integrar correctamente el sistema permitirá localizar de forma rápida y eficiente los documentos, así como facilitar las búsquedas y propiciar su actualización.

Respecto a las necesidades de formación profesional, la incorporación de temáticas al curriculum educativo en la formación de profesionales de la información y la documentación es un paso inevitable, considerando que otras disciplinas se caracterizan también por la actividad constante en el desarrollo de documentos personales y en el uso constante de TIC como parte fundamental del trabajo y de sus actividades personales cotidianas.

Un campo de acción profesional característico de las necesidades de administración de información personal sucede dentro de las universidades y centros de investigación, cuyo personal académico, se ha convertido en uno de los principales focos de atención dada su constante actividad al estar involucrados en procesos de generación y aplicación de conocimiento, además requieren mantener actualizados sus repositorios a fin de localizarlos de forma rápida y constante.

Este campo de acción profesional ofrece una tipificación clara de los documentos que se generan, ya que no se parte de la definición propia, sino de la proporcionada por las instancias que se dedican a los procesos de evaluación docente e investigativa.

$\mathrm{Si}$ el académico o investigador ha incorporado a su vida cotidiana la generación de documentos personales, entonces el dominio de las ciencias computacionales se hace inminente, demostrando para ello, la capacidad para ejecutar, acceder y evaluar la información, usando diversos mecanismos de control. 
La problemática que surge es de carácter técnico -al no tener conocimiento o habilidades para el uso de las TIC-, en este sentido, se espera que existan profesionales de la documentación capaces de conocer los mecanismos de administración de la información personal a través de tecnologías modernas, impulsando incluso a docentes e investigadores hacia la generación de nuevas publicaciones de forma constante y sistemática.

De esta forma, existen dos alternativas para acercarse al tema de la administración de la información personal: en la primera, vinculada a la autogestión, el propietario de los documentos desarrolla su propio proceso; y la segunda, relacionada con el reconocimiento de involucrar a profesionales de la información para que brinden esta clase de servicios.

Se presenta aquí la incorporación de una nueva tendencia profesional: la de gestor de información personal, que en opinión de Coughlan y Johnson (2008) deberá estar matizado por las siguientes características:

a) Mostrar capacidad para identificar una variedad de necesidades personales a fin de desarrollar alternativas compatibles que respondan a los requerimientos que se demanden, identificando aquellos que agreguen valor al proceso.

b) Demostrar una interacción creativa que permita la concesión de ideas y ofrezca alternativas en el diseño de herramientas de soporte para los propósitos que se demandan.

c) Retener y administrar ideas e información, mismas que ofrezcan un futuro potencial para integrar sistemas de información personal que localicen de forma eficiente los documentos demandados, y a su vez, propicien la generación de conocimiento.

d) Permitir la generación de ideas por parte del dueño de los documentos personales, respetando su ordenación original y propiciando la integración de sistemas de control.

e) Poseer conocimientos técnicos sobre lenguajes documentales, información semántica, sistemas de almacenamiento y recuperación de información, métricas de la información (Chernov, 2008), procesos de consultoría, TIC y desarrollo de proyectos, entre otros aspectos.

\section{CONCLUSIONES}

Una limitada e imprecisa documentación bibliográfica, electrónica y hemerográfica, exige a la comunidad disciplinar de la bibliotecología, ciencias de la información y ciencias de la documentación, acciones que pongan sobre la palestra de la discusión el tema de la información personal y su impacto en la era digital. 
Hasta ahora, las propuestas de procesos de gestión informativa personal están fundamentadas en metodologías simples, basadas en experiencias comunes y no necesariamente científicas (Bergman, Beyth-Maron y Nachmias, 2006).

Es indispensable crear una taxonomía que identifique los tipos de documentos personales y sus características ya que determinará en gran medida el diseño de procesos metodológicos precisos, científicamente aceptables y generalizables a múltiples contextos de aplicación.

Identificar los fundamentos conceptuales y metodológicos y diseñar los sistemas que permitan su gestión son dos de los asuntos pendientes que tendrá que superar el tema de la información personal en la sociedad del conocimiento.

Solucionar la no fragmentación dependerá de la calidad de los modelos y prototipos de gestión -diseñados ex profeso para resolver necesidades específicas-.

Los planes de estudio juegan un papel fundamental en la formación de recursos humanos, ofreciendo un perfil de egreso con la capacidad de afrontar los retos de la información personal, por tanto, el curriculum relacionado con las ciencias de la información y documentación tendrá que considerar una reforma que actualice y le de nuevos alcances ante los temas que surgen cotidianamente. Este nuevo perfil o enfoque curricular deberá orientarse hacia la capacidad diagnóstica del egresado que le permita diseñar propuestas y encontrar soluciones en su área de acción profesional.

\section{REFERENCIAS}

BARREAU, D. «Context as a Factor in Personal Information Management Systems», Journal of the American Society for Information Science, 1995, 46/5, 327-339.

BARREAU, D., Nardi, B. «Finding and Reminding: File organization from the Desktop», Association of Computing Machinery SIGCHI Bulletin, July 1995, 27/3,39-43.

BELLOTT I, V. et al. Taking email to task: the design and evaluation of a task management centered email tool. En: Proceeding of the SIGCHI conference on human factors in computing systems, New York: ACM Press, 2003, 345-352.

BERGMAN, O. et al. Personal Information Management. En: Proceedings of Conference of Human Factors in Computing Systems, CHI 2004, Vienna, Austria, April 24-29, 2004.

BERGMAN, O., Beyth.Marom, R., Nachmias, R. The project fragmentation problem in Personal Information Management. En: CHI 2006 Proceedings, April 22-27, 2006, Montreal, Canadá.

BOARDMAN, R. Improving Tool Support for Personal Information Management. Tesis Doctoral (Doctor de Filosofía) - University of London, 2004.

BOARDMAN R., Sasse, M. Angela. Stuff goes into the Computer and doesn't come out: A Crosstool Study of Personal Information Management. En: Proceedings of CHI2004. New York: ACM Press, 2004, pp. 583-590. 
BOARDMAN, R., Sasse, M. Angela; Spence, Bob. Life beyond the mailbox: A Cross-Tool Perspective on Personal Information Management. En: CSCW 2002 Workshop: Redesigning E-mail for the 21st Century, 2002.

BOARDMAN, R., Spence, Robert; Sasse, M. Angela. Too Many Hierarchies?: The Daily Struggle for Control of the Workspace. En: Proceedings of HumanComputer Interaction International. New Jersey: Lawrence Erlbaum Associates, 2003, pp. 616-620.

CHERNOV, Sergey et al. Evaluation Personal Information Management Using an Activity Logs Enriched Desktop Dataset. (http://pim2008.ethz.ch/paper/pim2008 chernov-etal.pdf). Consulta: 2-11- 2012.

COLLINS, Anthony; KAY, Judy. Collaborative personal information management with shared, interactive tabletops. En: Proceedings of CHI2008 Workshop: personal Information Management, Florence, Italy, April 5-10, 2008.

COUGHLAN, Tim; JOHNSON Peter. Personal Information Management for Creative Practitioners. En: Workshop: Personal Information Management, Florence, Italy, Proceedings of CHI2008, April 5-10, 2008.

DONG, X. A Platform for personal information management and integration. En: Proceedings of VLD 2005 PhD Workshop, August 29, 2005, Trondheim, Norway.

DONG, X., Halevy, A., Nemes, E., Sigurdssin, S. B., Domingos, P. SEMEX: Toward on-the-fly personal information integration. En: Proceedings of the International Workshop on Mining for and from the Semantic Web, Seattle, WA, 2004, pp. 4455.

FERRÁN-FERRER, N., PÉREZ MONTORO, M. «Gestión de información personal en usuarios avanzados en TIC», El Profesional de la Información, julio-agosto, 2009. 18/4, 365-373. DOI: 10.3145/epi.2009.jul.02.

GONÇALVEZ, Daniel; JORGE, Joaquim A. Now, it's Personal: Evaluating PIM Retrieval Tools. En: Workshop: personal Information Management, Florence, Italy, Proceedings of CHI2008, April 5-10, 2008.

JONES, W., TEEVAN, Jaime. Personal Information Management. Seattle, WA; London: University of Washington Press, 2007.

JONES, W. et al. Towards a unification \& integration of PIM support. Seattle, WA: University of Washington, 2008.

JONES, W. How is information personal? Seattle, WA: University of Washington, 2008.

KATIFORI, N. et al. Personal Ontology Creation and Visualization for a Personal Interaction Management System. En: Proceedings of CHI, 2008, Florence, Italy, April 5-10, 2008.

KHOO, C.S.G., LUYT, B., Ee, C., ORMAR, J., LIM, H.H., YONG, S. «How users organize Electronic files on their workstations in the office environment: a preliminary study of personal Information organisation behaviour»,. Information Research, 11/2, January, 2007. 
KOMNINOS, Andreas; BAILLIE, Lynne; BARRIE Peter. Holistic PIM: Managing Personal Information for a Nomadic Generation. En: Conference of Human Factors in Computing Systems, CHI 2008 Proceedings, Florence, Italia, April 5-10, 2008.

KRATKIEWICZ, G.; Mitchell, G. An adaptive semantic approach to personal information management. En: IEEE International Conference on System, Man and Cybernetics, October, 2004, 2/10-13, pp. 1395-1400.

LANSDALE, M. «The Psychology of Personal Information Management», Applied Ergonomics, 19/1, 1998, 55-66.

LÓPEZ YEPES, José, (Comp.) Diccionario enciclopédico de ciencias de la documentación. Madrid, España: Editorial Síntesis, 2004.

LOZANO TELlO, A. Ontologías en la Web Semántica. Ponencia presentada en I Jornadas de Ingeniería Web'01, Cáceres, España: Universidad de Extremadura, 2011.

MCCALLISTER, E., GRANCE, T., SCAREFONE, K. Recommendations of the National Institute of Standards and Technology. Gaithersburg, MD: NIST, 2010.

NARAYANAN, A., SHMATIKOV, V. «Privacy and security: myths and fallacies of "Personally Identifiable Information"», Communication of the ACM, 53/3, June, 2010. DOI: $10.1145 / 1743546.1743558$.

NAVARRO JIMÉNEZ, G. I. El Derecho a la protección de información personal en México. http://www.ordenjuridico.gob.mx/Congreso/2doCongresoNac/pdf/Navarro.pdf. Consulta: 2-11- 2012.

SAUEMANN, L., ELST, L., DANGEL, A. PIMO: a framework for representing personal information model. En: Proceedings of the International Workshop on Mining for and from the Semantic Web, Seattle, WA, 2004.

TEEVAN, J., JONES, W., BEDERSON, B. «Personal Information management», Comunication of the ACM, 49/1, January, 2006. 